\title{
An Optimal Broadcasting Algorithm for de Bruijn Network dBG(d,k)
}

\author{
Ngoc Chi Nguyen and Sungyoung Lee \\ Computer Engineering Department, Kyung Hee Univerity \\ 1, Seocheon, Giheung, Yongin, Gyeonggi 449-701 KOREA \\ \{ncngoc, sylee\}@oslab.khu.ac.kr
}

\begin{abstract}
Recent works have classified de Bruijn graph (dBG) based broadcasting algorithms into local broadcasting and arc-disjoint spanning trees based broadcasting. However, those algorithms can only work in binary dBG. In this paper, we investigate broadcasting in bidirectional $\mathrm{dBG}$ for a degree greater than or equal to two. A distributed broadcast algorithm for one-to-all broadcasting in the all port communication is proposed for $\mathrm{dBG}(\mathrm{d}, \mathrm{k})^{1}$.
\end{abstract}

\section{Introduction}

Broadcasting is one of the fundamental communication problems of interconnection networks. Some typical broadcasting applications are synchronizing different processors in a distributed computing network, and reconfiguring multiprocessor architecture. Recently, broadcasting problems on dBG have been investigated as local broadcasting[3] and arc-disjoint spanning trees based broadcasting[4][5].

However, the above can only work in a $\operatorname{dBG}(2, \mathrm{k})$ networks. Considering this limitation we intend to investigate broadcasting in bidirectional de Bruijn graph with a degree greater than or equal to two. A distributed broadcast algorithm is proposed for $\mathrm{dBG}(\mathrm{d}, \mathrm{k})$. Our study shows that the maximum time steps to finish broadcast procedure is $\mathrm{k}$ regardless of the broadcast originator, time complexity at each node is $0(3 \mathrm{~d} / 2)$, and no overhead happens in the broadcast message.

This paper is organized as follows: background is discussed in section 2, section 3 explains the algorithm and the paper is concluded in section 4 .

\section{Background}

The $\mathrm{dBG}$ graph denoted as $\mathrm{dBG}(\mathrm{d}, \mathrm{k})[1]$ has $\mathrm{N}=d^{k}$ nodes with diameter $\mathrm{k}$ and degree $2 \mathrm{~d}$. If we represent a node by $d_{0} d_{1} \ldots d_{k-2} d_{k-1}$, where $d_{j} \in 0,1, \ldots,(d-1)$, $0 \leq \mathrm{j} \leq(\mathrm{k}-1)$, then its neighbor are represented by $d_{1} \ldots d_{k-2} d_{k-1} p$ (L neighbors, by shifting left or $\mathrm{L}$ path) and $p d_{0} d_{1} \ldots d_{k-2}(\mathrm{R}$ neighbors, by shifting right or $\mathrm{R}$ path), where $p=0,1, \ldots,(d-1)$. Shift string of a node $\mathrm{A}$ is a binary string $(0$ for left shift and 1 for right shift) which represents path from originator to $\mathrm{A}$.

\footnotetext{
${ }^{1}$ This research was partially supported by ITRC project of Sunmoon University
} 
For simplest broadcasting mechanism, the originator initiates the process by making a "call" to other neighboring vertices in the graph informing them of the message. Subsequently, the informed vertices call their neighboring vertices and the process continues until all vertices in the graph are informed. Basically, this mechanism is like flooding phenomenon. Note that the interval during which a call takes place will be referred to as a time step or simply step. In flooding broadcasting (FB), level of a node $\mathrm{A}$ is the number of steps by which a message from originator reaches A (or shortest path length between $\mathrm{A}$ and originator).

\section{Broadcasting algorithm in $\mathrm{dBG}(\mathrm{d}, \mathrm{k})$}

By applying FB, we can easily obtain $\mathrm{k}$ as the maximum number of steps to finish broadcasting. However, message overhead is very high in FB. Thus, how to reduce message overhead (or letting each informed vertices call its uninformed neighbors only) in FB states the motivation for our algorithm. We assume that each packet sent to the other node must contain originator address, sender's level, a shift string of receiver and all calls take the same amount of time.

There are two cases of message overhead when an informed node $\mathrm{A}$ wants to inform node X. Case 1, node X has been informed already. Thus, X must have lower or equal level to $\mathrm{A}$. Case 2 , uninformed node $\mathrm{X}$ can be informed by nodes B,C,D, which have the same level as A, at the same time. For case 1, we need to compare the shortest-path length between $\mathrm{X}$ and $\mathrm{A}$ to originator. And $\mathrm{X}$ is informed by A if X level is higher than A's level and case 2 not happen. For case 2 , we have to define some conditions, based on these conditions only A or B or $\mathrm{C}$ or... inform X. The following theorems are proposed for calculating path length.

Theorem 1: given $p$ is shortest-path length between node a and $b$, the minimum length of matched strings between $a$ and $b$ is $k$ - $p(d B G(d, k))$.

Proof: as shown in [1], there are 3 types for determining shortest path (R,L; $\left.\mathrm{RL}, \mathrm{LR} ; R_{1} L R_{2}, L_{1} R L_{2}\right)$. The minimum matched string[2] can be obtained in type R,L among them. And length for this minimum matched string is k-p.

Theorem 2: path length between node $s$ and $d$ is $\min \left(2 s_{j}+s_{i}+d_{i}, 2 s_{i}+\right.$ $\left.s_{j}+d_{j}\right)$, where $s_{i}$ and $d_{i}$ are the left indices, and $s_{j}$ and $d_{j}$ are the right indices of matched string in $s$ and $d$ respectively.

Proof: path length $2 s_{j}+s_{i}+d_{i}, 2 s_{i}+s_{j}+d_{j}$ are for case $R_{s_{j}} L_{s_{j}+s_{i}} R_{d_{i}}$ and $L_{s_{i}} R_{s_{i}+s_{j}} L_{d_{j}}$ respectively . These cases are the general cases for 3 types presented in [1] (ex. if $s_{i}, s_{j}, d_{i}, d_{j} \neq 0$ then they become type $R_{1} L R_{2}$ and $L_{1} R L_{2}$ ).

To solve the above two cases of message overhead, a Boolean valued function SPL is proposed. SPL has inputs: originator $\mathrm{S}$, current node $\mathrm{P}$, neighboring node $\mathrm{X}$, current level $\mathrm{n}$ (level of $\mathrm{P}$ ), shift string $\mathrm{Q}\left(q_{0} q_{1} q_{2} \ldots q_{z-1}\right.$, length $\left.\mathrm{z} \leq \mathrm{k}\right)$ (from $\mathrm{S}$ to $\mathrm{X}$ through $\mathrm{P}$ ). Fig. 1a shows SPL algorithm. Step 1,2,3 solve message overhead of case 1 . Step 1 is a result of theorem 1 . Step 4,5,6 solve case 2 message overhead. In case 2 , we have several shortest paths from $\mathrm{S}$ to $\mathrm{X}$. One shortest path must be chosen based on the following conditions:

- The shortest path corresponds with the shortest matched string of S and $\mathrm{X}$ (step 5). 


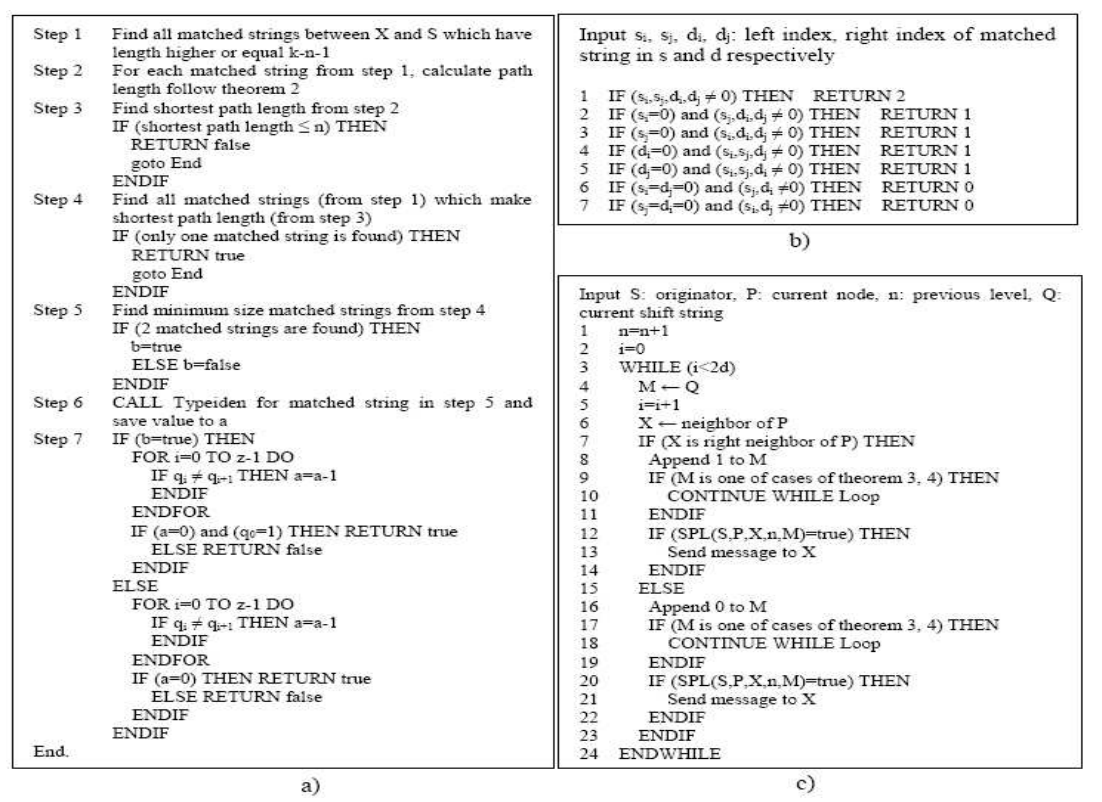

Fig. 1. a)SPL function algorithm; b)Typeiden function algorithm; c)Broadcasting algorithm for $\mathrm{dBG}(\mathrm{d}, \mathrm{k})$.

- In the case, there exist 2 shortest path from the first condition. Then, shortest path which begin with shifting right is chosen. (step 6)

Step 7 compares shift string Q to the condition gotten from step 5 and 6 to determine whether $\mathrm{X}$ should be informed or not.

Example 1: in $\mathrm{dBG}(3,10)$, given input S: 0012111001, P: 0111012110, $\mathrm{n}=7$, $\mathrm{X}: 1110121100, \mathrm{Q}=01111100$. By applying SPL, we have

Step 1: find all matched strings[2] which have length higher or equal 10-7$1=2$. These strings are 11, 111, 1110, 01, 012, 0121, 01211, 110, 1100 .

Step 2: path lengths for strings in step 1 are 12, 10, 8, 14, 12, 10, 8, 13, 11.

Step 3: shortest path length is 8 .

Step 4: matched string, which make shortest path length 8, are 1110, 01211.

Step 5: minimum size string from step 4 is $1110, \mathrm{~b}=$ false.

Step 6:Typeiden(input $s_{i}=0, s_{j}=6, d_{i}=4, d_{j}=2$ ) $\rightarrow$ returned value: $1, \mathrm{a}=1$.

Step 7: there are 2 places in $\mathrm{Q}$ in which two adjacent bits are different $\rightarrow \mathrm{a}=-$ $1 \neq 0$. Consequently, $\mathrm{X}$ is an uninformed node (step 3,8>n), but it isn't informed by $\mathrm{P}$ (message overhead case 2) due to our priority given in step 5 and 6 .

If we apply SPL for all $2 \mathrm{~d}$ neighbors of one node, then it cost $0(2 \mathrm{~d})$ for running our algorithm. The following theorems reduce from $0(2 \mathrm{~d})$ to $0(1.5 \mathrm{~d})$. Following are some notations used, where $\mathrm{T}$ is the previous shifting string.

$\mathrm{R} \leftrightarrow \mathrm{T}$ : total number of right shift in $\mathrm{T}>$ total number of left shift in $\mathrm{T}$

$\mathrm{L} \leftrightarrow \mathrm{T}$ : total number of left shift in $\mathrm{T}>$ total number of right shift in $\mathrm{T}$

Theorem 3: by shifting $R L R / L R L$, results are duplicate with shifting $R / L$. 
Proof: given a node $a_{0} a_{1} \ldots a_{n-1}$. By shifting RLR in $\mathrm{dBG}(\mathrm{d}, \mathrm{k})$, we have $a_{0} a_{1} \ldots a_{n-1} \rightarrow \alpha a_{0} a_{1} \ldots a_{n-2} \rightarrow a_{0} a_{1} \ldots a_{n-2} \beta \rightarrow \gamma a_{0} a_{1} \ldots a_{n-2}, 0 \leq \alpha, \beta, \gamma<\mathrm{d}$.

Substitute $\alpha$ for $\gamma \rightarrow \gamma a_{0} a_{1} \ldots a_{n-2} \equiv \alpha a_{0} a_{1} \ldots a_{n-2}$.

By proving similarly for case LRL, theorem 3 is proved.

Theorem 4: if $R \leftrightarrow T / L \leftrightarrow T$, results provided by next shift $L R / R L$ are duplicate.

Proof: assume the beginning node is $a_{0} a_{1} \ldots a_{n-1}$. For case $\mathrm{R} \leftrightarrow \mathrm{T}$, we have the following cases:

- $T=R_{u} L_{v} R_{w}, T=L_{u} R_{v} R w, T=L_{u} R_{v}$. By shifting LR, we have shift string $R_{1} L_{1} R_{2} L_{2}$ or $L_{1} R_{1} L_{2} R_{2}$, which are not existed for shortest path (as shown in Lemma 1 of [1]).

- $T=R_{u} L_{v}(\mathrm{u}>\mathrm{v})$. By shifting $\mathrm{R} \mathrm{u}$ times and $\mathrm{L} \mathrm{v}$ times respectively, we have $a_{0} a_{1} \ldots a_{n-1} \rightarrow \beta_{u-1} \ldots \beta_{1} \beta_{0} a_{0} a_{1} \ldots a_{n-u-1} \rightarrow \beta_{u-v-1} \ldots \beta_{1} \beta_{0} a_{0} a_{1} \ldots a_{n-u-1} \delta_{0} \delta_{1} \ldots \delta_{v-1}$ where $0 \leq \beta_{i}, \delta_{j}<\mathrm{d}, 0 \leq \mathrm{i}<\mathrm{u}, 0 \leq \mathrm{j}<\mathrm{v}$. By shifting LR we have,

$\beta_{u-v-1} \ldots \beta_{0} a_{0} \ldots a_{n-u-1} \delta_{0} \ldots \delta v-1 \rightarrow \beta_{u-v-2} \ldots \beta_{0} a_{0} \ldots a_{n-u-1} \delta_{0} \ldots \delta v \rightarrow$

$\gamma \beta_{u-v-2} \ldots \beta_{0} a_{0} \ldots a_{n-u-1} \delta_{0} \ldots \delta v-1(\mathrm{~K})$

Substitute $\gamma(0 \leq \gamma<\mathrm{d})$ for $\beta_{u-v-1} \rightarrow \mathrm{K}$ is duplicate.

- $\mathrm{R}=R_{u}$. Shift string $R_{u} L R$ makes duplicate as shown in theorem 3 .

By proving similarly to case $\mathrm{L} \leftrightarrow \mathrm{T}$, we prove theorem 4 .

As a result, broadcasting algorithm is proposed as shown in fig. 1c.

Theorem 5: in the worst case, time complexity for our broadcasting algorithm is $0(1.5 d)$.

Proof: probability for theorem 3 happening is $25 \%$, and for theorem 4 is less than $25 \%$. Therefore, the probability for CONTINUE command (line 10, 18 fig. $1 \mathrm{c}$ ) happening is $25 \%$. So, theorem 5 is proved.

\section{Conclusion}

We have presented a distributed broadcasting algorithm for $\mathrm{dBG}(\mathrm{d}, \mathrm{k})$, which requires $\mathrm{k}$ steps to finish broadcasting process, time complexity at each node is $0(3 \mathrm{~d} / 2)$ and no message overhead during broadcasting. Therefore, the algorithm can be considered feasible for broadcasting in the real interconnection network which is built based on de Bruijn graph $\mathrm{dBG}(\mathrm{d}, \mathrm{k})$.

\section{References}

1. Zhen Liu, Ting-Yi Sung, "Routing and Transmitting Problem in de Bruijn Networks" IEEE Trans. on Comp., Vol. 45, Issue 9, Sept. 1996, pp 1056 - 1062.

2. Alfred V. Aho, Margaret J. Corasick, "Efficient String Matching: An Aid to Bibliographic Search", Comm. of the ACM, Vol. 18 Issue 6, June 1975.

3. A.H.Esfahanian, G. Zimmerman, "A Distributed Broadcast Algorithm for Binary De Bruijn networks", IEEE Conf. on Comp. and Comm., March 1988.

4. E.Ganesan, D.K.Pradhan, "Optimal Broadcasting in Binary de Bruijn Networks and Hyper-deBruijn Networks", IEEE Symposium on Parallel Processing, April 1993.

5. S.R.Ohring, D.H.Hondel, "Optimal Fault-Tolerant Communication Algorithms on Product Networks using Spanning Trees", IEEE Symp. on Parallel Processing, 1994. 